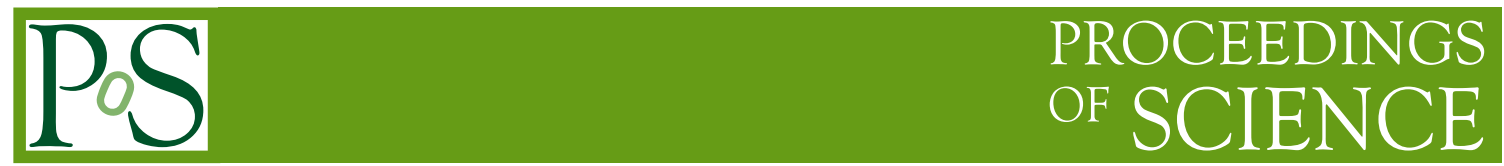

\title{
Calculational Techniques in Particle Theory
}

\section{Lorenzo Tancredi ${ }^{a, b}$}

${ }^{a}$ Physik Department, Technische Universität Mü̈chen, James-Franck-Straße 1, 85748 Garching, Germany

${ }^{b}$ Rudolf Peierls Centre for Theoretical Physics, Clarendon Laboratory, Parks Road, Oxford OX1 3PU, UK

E-mail: lorenzo.tancredi@tum.de

In this contribution, I review some of the latest advances in calculational techniques in theoretical particle physics. I focus, in particular, on their application to the calculation of highly non-trivial scattering processes, which are relevant for precision phenomenology studies at the Large Hadron Collider at CERN.

*** The European Physical Society Conference on High Energy Physics (EPS-HEP2021), *** *** 26-30 July $2021 * * *$

*** Online conference, jointly organized by Universität Hamburg and the research center DESY *** Preprint Number: TUM-HEP-1370/21 


\section{Introduction, SM and Precision Physics}

The discovery of the Higgs Boson at the CERN LHC in 2012 [1,2] has been a milestone in particle physics, providing firm ground for the impressive theoretical edifice of the Standard Model of Particle Physics. Despite the fact that, with the Higgs discovery, the full matter content of the Standard Model has now been observed, there are still many unanswered questions about its theoretical foundations. One of the most pressing issues is the origin and structure of the Yukawa interactions between the Higgs boson and the fermions (quarks and leptons). Among the other things, the way the Higgs couples to fermions, in particular the patterns of its interactions with the first family of quarks and leptons, determines the masses of those particles and, ultimately, it is the reason why chemistry has allowed for the existence of life as we know it.

Until recently, the only indication that we had of the Higgs coupling to fermions was indirect, namely through the observation of its production in gluon fusion and decay to photons; in the Standard Model, both are in fact loop-induced processes and happen through the coupling of the Higgs to the heavy top quark. A real breakthrough took place in 2018, when both ATLAS and CMS observed, for the first time, the production of a $t \bar{t}$ pair in association with a Higgs boson [3, 4],t $\bar{t} H$. This was the first direct observation of the coupling of the Higgs boson with top quarks. After this result, more have followed, in particular confirming that the Higgs couples, roughly as expected, to the third family of quarks and leptons, see for example recent results in [5].

But let us keep our focus on $t \bar{t} H$, which provides a perfect example of the challenges that the experimental results obtained at the LHC are posing to the theory community. Already in their first measurements in 2018, ATLAS and CMS could get a good estimate of the relevant Yukawa coupling, with an overall precision around $\sim O(15-20 \%)$. This measurement was performed by analysing a small fraction of the full expected data-set that will be produced at the LHC. Indeed, compared the total amount of data that will be collected starting with Run 3 and continuing through the High-Luminosity phase (HL-LHC), only $5 \%$ of the total collisions expected have already been delivered. A naive estimation would suggest that the statistical error on these measurements could go down of a factor of 6 at HL-LHC, bringing it down to the few percent level.

Keeping up with such precision on the theoretical side is very challenging. The theoretical modelling of high-energetic collisions at the LHC is in fact notoriously difficult, due to the interplay of physics at very different energy scales. Here, the existence of (collinear) factorisation theorems in Quantum Chromodynamics (QCD) allows us to separate non-perturbative long-range (low-energy) physics from perturbative short-range (high-energy) physics effect in a consistent way, such that corrections to the factorised picture are expected to be of the order of $O\left(\Lambda_{Q C D} / Q\right)$, where $\Lambda_{Q C D}$ is the typical non-perturbative QCD scale and $Q$ is the scale of the process considered. At the scales of LHC collisions such corrections can be expected to be at the percent level, which justifies our ambition to try and describe theoretically scattering processes in QCD with a comparable precision. Special care should be used, anyways, as factorisation theorems are only rigorously proven for a very limited number of processes with relatively simple kinematics.

Even assuming the correctness of the factorised picture for complex final states as $t \bar{t} H$, a lot of effort has then to be devoted on the precise determination of the non-perturbative building blocks that make up this picture, most notably the parton distribution functions; in addition, the precise simulation of realist icprocesses, including parton showers, hadronisation, fragmentation, detector 
response etc, must also be understood. Only by dedicating an equal effort to understanding all these pieces of the puzzle, can we dedicate our attention to study high-energetic (hard) collisions of fundamental partons with percent level precision. In what follows, I will report on many new results and fascinating new methods developed to the aim of improving our understanding of fixed-order calculations for complex LHC processes.

\section{Fixed-order calculations for LHC processes}

Quantum Field Theory (QFT) provides us with a set of well defined rules which allow us, under certain assumptions, to improve consistently our prediction for a given scattering process. Schematically, in QFT we evaluate cross-sections (and more in general differential observables) by first computing so-called scattering amplitudes $\mathcal{A}$, which provide the probability amplitudes for the partonic scattering to take place. Very schematically, an observable $O$ can be computed as

$$
\sigma_{O} \sim \int|\mathcal{A}|^{2} d \mathrm{PS}
$$

where $d \mathrm{PS}$ symbolises the integration measure over the relevant phase-space that defines the observable considered.

The scattering amplitudes $\mathcal{A}$ are complex functions of the kinematics and of the spins and polarisations of the relevant particles, and can be computed perturbatively in QFT by an expansion in Feynman diagrams. Importantly, in order to increase the perturbative order, we typically need to compute Feynman diagrams with more loops and higher multiplicities, or, in jargon, with more legs. While these calculations can be extremely difficult, the many results obtained in the past years have revealed unexpected regularities in the final analytical expressions for the scattering amplitudes, which in turn have fuelled the study of their mathematical properties from a general perspective. In what follows, I will review some of the most recent developments in this field.

\section{New ideas for Scattering Amplitudes}

The standard text-book approach to compute scattering amplitudes goes through the enumeration and evaluation of the relevant Feynman diagrams that contribute to the scattering process at the perturbative ordered considered. While straightforward in principle, computing Feynman diagrams can quickly become extremely cumbersome. First of all, there is clearly an obvious combinatorial reason why we rightfully expect scattering amplitudes to increase in complexity with the number of loops and legs. Nevertheless, it has been observed over and over again in all scattering amplitudes calculations, that the apparent complexity which one has to deal with in the intermediate stages of a calculation, is often orders of magnitude worse than the real complexity of the final, physical results. The main reason why this happens, is the way we perform perturbative calculations in QFT: by insisting in using off-shell Feynman diagrams, which carry multiple redundancies due to the virtual particles running in the loops and to gauge freedom, we hide the simplicity of on-shell, gauge invariant scattering amplitudes.

In order to review the most recent developments in this field, I find it useful to separate the types of problems that one encounters when performing these calculations, into three separate categories: 
1. Obtaining the integrand, namely the expression which needs to be integrated.

2. Reducing the integrand to master integrals.

3. Computing these master integrals either numerically or analytically.

The issues which stem from the three points above are clearly connected and a strict separation among them is of course impossible. I will nevertheless use this simplifying categorisation to summarise the recent advances in the different directions.

\subsection{Obtaining the integrand}

As we discussed above, Feynman diagrams provide us with a bullet-proof technique which allows us to obtain the integrand for any scattering amplitude in any (perturbative) Quantum Field Theory. This approach is plagued by a quickly growing combinatorial complexity and in recent years a lot of effort has been devoted to understand how to construct the integrand avoiding a blind generation and manipulation of all Feynman diagrams. By generalising on-shell techniques valid at tree-level $[6,7]$ and one-loop [8-11], many ideas have been developed to try and avoid Feynman diagrams entirely also at higher loops, and build integrands only from on-shell, gauge invariant data [12-15]. In the same spirit, but from a different point of view, interesting results have also recently been obtained on extending recursion techniques (based on Feynman diagrams!) to construct two-loop integrands efficiently starting from lower loop amplitudes [16-18]. These developments are very promising, and they have made it possible to compute various non-trivial amplitudes, in particular in the very special all-plus configuration [19,20]. Nevertheless, none of these techniques is still mature enough to provide a workable alternative to Feynman diagrams for generic helicity configurations and in non-supersymmetric theories.

So while it does not seem likely that we will be able to get rid of Feynman diagrams for QCD calculations in the very near future, an interesting question is how we can improve the way we manipulate the Feynman diagrams, in order to obtain the sought for integrand in the most efficient and compact form possible. The main goal of these manipulations is to resolve the Dirac algebra and to express the Feynman diagrams in terms of loop-independent Lorentz structures, which multiply loop-dependent scalar Feynman integrals. The latter are simpler to compute and we will discuss various techniques to do this in the next two sections.

One standard approach to extract scalar Feynman integrals from scattering amplitudes in terms of Feynman diagrams, is the so-called projector method. Schematically, this works by decomposing the scattering amplitude, tentatively non-perturbatively, in terms of so-called tensors and invariant form factors $F_{j}$

$$
\mathcal{A}=\sum_{j=1}^{N} F_{j} T_{j} .
$$

With a slight abuse of language, we call "tensors" $T_{j}$ are all Lorentz covariant structures that can be built and which are consistent with the symmetries of the problem. The $T_{j}$ include the relevant polarisation vectors / spinors that contract the Lorentz indices and transform therefore only under the corresponding Little Group. Once we have the decomposition in eq. (1), we can derive projector 
operators which single out the corresponding form factors. Again schematically, we define the projector $P_{k}$ as

$$
P_{k}=\sum_{j=1}^{N} c_{j}^{(k)} T_{j}^{\dagger}, \quad P_{k} \cdot \mathcal{A}=F_{k},
$$

where the action $P_{k} \cdot \mathcal{A}$ is defined by summing over all spins and helicities of the external particles. This construction is usually done in the so-called conventional dimensional regularisation scheme (CDR), ie treating all momenta, internal and external, as $d$-dimensional. While the method was successfully applied to many $2 \rightarrow 1$ and $2 \rightarrow 2$ calculations up to two and three loops, it turns out to scale very badly with the increase of the number of external particles and tends to become impractical already for $2 \rightarrow 3$ processes, see for example [21].

This complexity stems from the desire of using this method in CDR. Nevertheless, it was recently realised that if one is interested in computing the "helicity amplitudes" for the process in the 't Hooft-Veltman regularisation scheme (tHV) this method can be simplified extremely [22, 23]. In fact, one can exploit the fact that many of the tensors $T_{j}$ in eq. (1) become linearly dependent when the dimensionality of the external states is equal to four, in order to block-diagonalise the projector matrix and define a subset of physical tensors whose number matches one-to-one that of the different helicity amplitudes. As it should be, the number of helicity amplitudes provides then an upper bound to the number of the independent tensors required. Recently, these ideas have been applied successfully to simplify the calculation of the three-loop corrections to $2 \rightarrow 2$ scattering in QCD [24, 25]. A different approach was also developed, where explicit representations for the polarisation vectors and spinors are used in order to single out the different helicity configurations from the scattering amplitudes [26] Both approaches have been successfully applied to various $2 \rightarrow 3$ scattering processes [27-29].

A different approach to extract integrands in QCD and which can be applied also on a Feynman diagram representation of the scattering amplitude is integrand decomposition [30-33]. Mixing integrand reduction with unitarity based techniques, has also been a very successful strategy [3436], and has allowed to obtain very non-trivial results for a large number of $2 \rightarrow 3$ scattering amplitudes [37-44]. More on the other crucial developments that have made these calculations possible will be discussed in the next paragraph.

\subsection{Reducing the integrand to master integrals}

Obtaining the integrand for a complicated scattering amplitude in QCD (or in general in the Standard Model) is only the very first step towards its calculation. Typically, after the application of any of the techniques described above, integrands for two or three-loop problems in realistic theories are expressed in terms of a large number of scalar integrals, which can go from tens of thousands up to many millions. Clearly, it is out of question to compute each of these integrals on its own. Luckily, the vast majority of these integrals are not linearly independent, and can instead be expressed as linear combinations of a (relatively) small number of so-called master integrals. While there are various ways to achieve this decomposition, the algorithmic use of integration-by-parts identities (IBPs) [45, 46], through the by-now famous Laporta algorithm [47], has definitely stand out as one of the most powerful and versatile techniques. IBPs have been applied to a multitude of 
problems in QCD, from two-point functions up to five loops [48, 49], to five point functions at two loops, which I will say more about later.

Unfortunately, since the Laporta algorithm maps the problem of reduction to master integrals to the symbolic solution a large system of linear equations, its blind application to solve complex multiloop problems can quickly become unfeasible even on the largest computers, due to the swell of the intermediate algebraic expressions. While it is fair to say that the impressive increase of computational resources available to theoretical particle physicists has played an important role in making many outstanding calculations finally possible, the past years have witnessed the emergence of revolutionising ideas that have lead to a breakthrough in the field. A first important realisation was the fact that out of the thousands or millions of IBPs produced in the standard Laporta approach, only a fraction is really relevant for the reduction of the physical scattering amplitudes. This happens for two reasons. First of all, because many of the identities are simply not independent and can be thrown away. This was known for a long time but was, to the best of my knowledge, first fully exploited only recenly in the public code Kira [50]. Secondly, one can devise algorithms, based on algebraic geometry, in order to "slice" through the space of IBP identities and select among those, only the ones that are so-called unitarity compatible [51-53]. This is a way to say, that we would like to select only those identities that never introduce integrals with spurious double propagators, which do not appear in scattering amplitudes, except in very special degenerate configurations. A lot of work has been done in this direction, leading to important developments for the simplifications of the systems of integration by parts identities.

In parallel to this, a large impact has come from the introduction of methods based on Finite Fields reconstruction. Indeed, Finite Fields have been used in algebra systems for decades to simplify computationally expensive operations as the computation of the great common divisor (gcd) of two polynomials. The breakthrough in particle physics has come from the realisation that, due to the simplicity of the final physical results, one can use Finite Fields reconstruction techniques in order to perform the chain of operations that express the finite remainder of complicated scattering amplitudes in terms of independent building blocks, without ever having to manipulate complicated intermediate expressions [54, 55]. It turns out, in fact, that by repeating the numerical evaluation of the scattering amplitudes many times over finite fields, one can uniquely reconstruct the analytic form of the rational functions that appear in the final result. We stress that the strength of this approach resides in the fact that the final physical result is often orders of magnitudes simpler than the intermediate steps of the calculation. In the past few years this technology has been adopted and improved upon by many groups, both in public [56-59] and private codes.

Together with Finite Field reconstruction techniques, also worth of mention is the introduction of new algorithms for the decomposition of multivariate rational functions into partial fractions [34, $62,63]$. Indeed, for a long time partial fraction decomposition has been the standard approach to simplify complicated rational functions showing up into QFT calculations. Until few years ago, nevertheless, the advent of larger computers and better gcd algorithms, had initiated a shift in the particle physics community to prefer more economic gcd-based representations for the final results. Only very recently it has been rediscovered that the rational functions which show up in scattering amplitudes are very poorly represented when put under great common divisor, also due to the 
presence of high poles in their denominators. Take for example the very compact function

$$
R(a, b, c, d)=\frac{1}{(a-b)^{6}}-\frac{1}{(c-d)^{5}} .
$$

By forcing it into a gcd representation, we obtain a much more complicated expression

$$
\begin{aligned}
& R(a, b, c, d)=- \\
& \frac{a^{6}-6 a^{5} b+15 a^{4} b^{2}-20 a^{3} b^{3}+15 a^{2} b^{4}-6 a b^{5}+b^{6}-c^{5}+5 c^{4} d-10 c^{3} d^{2}+10 c^{2} d^{3}-5 c d^{4}+d^{5}}{(a-b)^{6}(c-d)^{5}}
\end{aligned}
$$

Inverting the gcd is in general non-trivial, as partial fraction decompositions are not unique when many variables are involved and, in particular, different choices for the ordering of the variables with respect to which the decomposition is done, can lead to various issues as, for example, the appearance of undesired spurious poles. The new algorithms that I hinted to above, which are based on well known mathematics [60,61], make it possible to avoid the appearance of spurious poles and, to obtain compact and numerically very stable representations for complicated scattering amplitudes. Notably, in this way extremely compact representations have been obtained also for non-leading color amplitudes which involve also complicated non-planar Feynman integrals [29, 43, 62].

\subsection{Computing the master integrals}

In the previous two paragraphs, we have discussed about two major issues connected to the algebraic complexity of scattering amplitudes: how to manipulate the large expressions that emerge from multiloop / multileg Feynman integrals and how to efficiently decompose them in terms of independent master integrals. In our discussion, we have neglected a very non-trivial aspect of the whole story, namely the computation of the master integrals themselves in terms of special functions. Usually, it is indeed only when the finite remainder of a scattering amplitude in $d=4$ dimensions is expressed in terms of well defined special functions, that their simplicity becomes manifest.

Integration is notably a very non-trivial operation and an impressive effort has been devoted to the developments of efficient integration techniques for multiloop Feynman integrals. Given their complexity, the first obvious question one has to answer is whether it makes sense to attempt to evaluate Feynman integrals analytically, or if a flexible numerical approach are bound to be more efficient in the long run. Many advances have happened in both directions and, while it would be impossible to summarise all papers which are worth being mentioned, I will do my best to point out a couple of results which are, in my very personal opinion, particularly interesting.

\section{Analytical techniques}

A lot of progress has been achieved in analytical integration methods after the realisation that Feynman integrals in dimensional regularisation are often naturally expressed in terms of special classes of functions, that go under the general name of (Chen) iterated integrals [64].

Before going into the details of the results obtained, it is important to clarify what do we mean when we say that a result is "analytic". Usually, when we talk about an analytic result we imagine it to be written in terms of elementary "known functions", for example exponentials, logarithms, etc. 
As a matter of fact, except for some notable exceptions, Feynman integrals cannot be expressed in terms of elementary functions, and instead more general "special functions" are required, some of which might not have a name yet. The main problem is then to classify which types of functions are relevant and to study their properties. If we define a new class of functions, I think it is fair to say that a result written written in terms of them can be defined to be analytic as long as:

1. one has full analytic control on the functional relations among them.

2. one has full control over their numerical evaluation, through for example series expansions.

An especially celebrated class of special functions which fulfils these requirements and which has found large applicability in the context of Feynman integrals calculation, are the so-called multiple polylogarithms (MPLs). They are defined as iterated integrals over linear rational kernels with single poles

$$
G\left(a_{1}, \ldots, a_{n} ; x\right)=\int_{0}^{x} \frac{d y}{y-a_{1}} G\left(a_{2}, \ldots, a_{n} ; y\right), \quad G(x)=1, \quad G(\underbrace{0, \ldots, 0}_{n} ; x)=\frac{1}{n !} \log ^{n}(x),
$$

see $[65,66]$ and references therein.

A revolution in direct integration methods, based for example on the well known FeynmanSchwinger parametrisation, has happened with the establishment of the criterion of linear reducibility [67] for Feynman integrals and the development of algorithms to exploit it to compute Feynman integrals in terms of MPLs [68]. Many important calculations have been performed thanks to this insight, recent examples are various form factor integrals up to four loops [69] and $2 \rightarrow 2$ scattering amplitudes with masses [70].

Equally impressive analytic results have been obtained by the use of the by now renown method of differential equations [71-73], augmented by the choice of a canonical basis of integrals with unit leading singularities, as first proposed in [74]. This has made it possible to obtain analytical results in terms of special classes of Chen iterated integrals for all master integrals relevant to compute five-point two-loop massless scattering amplitudes. The relevant special functions have been dubbed "pentagon functions" [75]. More recently, also the first results for the corresponding pentagon functions for five-point functions with one off-shell leg been obtained [44, 76]. For these classes of problems, also results in terms of multiple polylogarithms have been obtained [77, 78], using a variant of the differential equations method [79]. I consider these results some of the latest successes of the analytic approach. Both criteria enumerated above (ie control over functional relations and numerical evaluation) are met by these results, which allowed various group to use them to obtain compact and stable expressions for all massless $2 \rightarrow 3$ processes, as discussed above. Very importantly, thanks to the newly obtained results for these scattering amplitudes, the first phenomenological studies of $2 \rightarrow 3$ processes in NNLO QCD have started to appear [80-83].

Before closing the discussion on the analytic methods, it is important to mention the efforts that are being made to extend our understanding of special functions beyond multiple polylogarithms. This line of research has been fuelled by a new geometrical understanding of iterated integrals of rational functions defined on increasingly complicated Riemann surfaces, the first example of which are the so-called elliptic polylogarithms. This line of research has sparked a lot of interest from different parts of the community, which would be impossible to summarise in few lines. Some example of recent work in this direct can be found in [84-90] and references therein. 


\section{(Semi-)Numerical techniques}

Finally, progress has been made also in the context of new numerical methods for the calculation of multiloop, multileg Feynman integrals. Clearly, numerical methods have an advantage on analytical ones, as they do not require a study and classifications of the special functions that can appear in the result. On the other hand, devising general numerical methods for the calculation of Feynman integrals is very non trivial, first of all because Feynman integrals are divergent both in the UV and the IR. This requires to write general procedures to isolate the poles and regularise the integrals. Even when this is done, a numerical evaluation in a physical region in Minkowski kinematics requires to find a proper contour deformation to avoid all the poles on the real integration domain. These poles are the origin of the discontinuities of the scattering amplitudes and it is crucial to pick the right integration contour in order to have well-defined results on the physical Riemann sheet. Worth of mention is definitely the impressive progress in direct numerical integration of Feynman integrals using the sector decomposition method [91], which has made it possible to compute various two-loop scattering amplitudes for $2 \rightarrow 2$ processes mediated by massive quarks $[92,93]$.

More recently, also a different semi-numerical approach has become increasingly popular to solve complicated multiloop Feynman integrals. The idea goes back to the well-known Frobenius method for the numerical solution of differential equations as series expansions close to regular singular points. In fact, one can use the (analytical) differential equations satisfied by the Feynman integrals, and use them to obtain fast converging series expansions for the integrals, which can in turn be used for their numerical evaluation [94, 95]. This method has been successfully applied, in various forms, to some non-trivial $2 \rightarrow 2$ and $2 \rightarrow 3$ two-loop Feynman integrals [96, 97].

An interesting question is then, with respect to which variable does it make sense to differentiate in order to get differential equations that are easy to solve numerically and automatically, with as few user input as possible. A possible answer has recently been provided in [98], where it has been proposed to differentiate with respect to auxiliary complex masses, say generically $m_{\text {aux }}$, suitably placed on all or only some of the propagators. This method has two advantages: first, one can obtain all boundary conditions at a value of the auxiliary masses going to infinity, from the algorithmic procedure of the large mass expansion. Second, since one integrates along the imaginary axis, one is usually far from singularities that are confined on the real axis and one needs to perform fewer steps in the numerical solution of the differential equations in order to transport the result from the boundary condition to the physical values of $m_{\text {aux }} \rightarrow 0$. As it is easy to imagine, there are some subtleties in how one recovers the limit $m_{\text {aux }} \rightarrow 0$, as in general Feynman integrals develop IR singularities when masses go to zero. Modulo these subtleties, this method can be very powerful and it has been applied already to the calculation of some non-trivial scattering amplitudes, see for example [99-102].

\section{Conclusions}

In conclusion, the last two decades have witnessed impressive developments in our understanding of the mathematics of Feynman integrals and how this mathematics plays a role to simplify the calculation of complicated scattering amplitudes. Thanks to these developments, results for very non-trivial scattering amplitudes at two and three loops have been obtained, which were entirely out 
of reach till few years ago. These have in turn made it possible to push the boundaries of precision calculations at the LHC to the NNLO level, reaching the few percent level precision for many interesting processes and observables. A plethora of new possibilities for precision phenomenological studies are opening, and possibly many other will open in the next few years, contributing to make the physics program at the LHC a success.

\section{Acknowledgements}

This work has been supported by the Excellence Cluster ORIGINS funded by the Deutsche Forschungsgemeinschaft (DFG, German Research Foundation) under Germany's Excellence Strategy - EXC-2094 - 390783311, by the ERC Starting Grant 949279 HighPHun and by the Royal Society grant URF/R1/191125.

\section{References}

[1] ATLAS collaboration, G. Aad et al., Observation of a new particle in the search for the Standard Model Higgs boson with the ATLAS detector at the LHC, Phys. Lett. B 716 (2012) 1-29, [1207. 7214].

[2] CMS collaboration, S. Chatrchyan et al., Observation of a New Boson at a Mass of $125 \mathrm{GeV}$ with the CMS Experiment at the LHC, Phys. Lett. B 716 (2012) 30-61, [1207.7235].

[3] ATLAS collaboration, M. Aaboud et al., Observation of Higgs boson production in association with a top quark pair at the LHC with the ATLAS detector, Phys. Lett. B $\mathbf{7 8 4}$ (2018) 173-191, [1806.00425].

[4] CMS collaboration, A. M. Sirunyan et al., Observation of $\mathrm{t} \overline{\mathrm{t}} H$ production, Phys. Rev. Lett. 120 (2018) 231801, [1804.02610].

[5] ATLAS collaboration, A combination of measurements of Higgs boson production and decay using up to $139 \mathrm{fb}^{-1}$ of proton-proton collision data at $\sqrt{\mathrm{s}}=13 \mathrm{TeV}$ collected with the ATLAS experiment, .

[6] R. Britto, F. Cachazo and B. Feng, New recursion relations for tree amplitudes of gluons, Nucl. Phys. B 715 (2005) 499-522, [hep-th/0412308].

[7] R. Britto, F. Cachazo, B. Feng and E. Witten, Direct proof of tree-level recursion relation in Yang-Mills theory, Phys. Rev. Lett. 94 (2005) 181602, [hep-th/0501052].

[8] Z. Bern, L. J. Dixon, D. C. Dunbar and D. A. Kosower, One loop n point gauge theory amplitudes, unitarity and collinear limits, Nucl. Phys. B 425 (1994) 217-260, [hep-ph/9403226].

[9] Z. Bern, L. J. Dixon, D. C. Dunbar and D. A. Kosower, Fusing gauge theory tree amplitudes into loop amplitudes, Nucl. Phys. B 435 (1995) 59-101, [hep-ph/9409265]. 
[10] G. Ossola, C. G. Papadopoulos and R. Pittau, Reducing full one-loop amplitudes to scalar integrals at the integrand level, Nucl. Phys. B 763 (2007) 147-169, [hep-ph/0609007].

[11] R. K. Ellis, Z. Kunszt, K. Melnikov and G. Zanderighi, One-loop calculations in quantum field theory: from Feynman diagrams to unitarity cuts, Phys. Rept. 518 (2012) 141-250, [1105.4319].

[12] S. Badger, H. Frellesvig and Y. Zhang, A Two-Loop Five-Gluon Helicity Amplitude in QCD, JHEP 12 (2013) 045, [1310. 1051].

[13] S. Badger, G. Mogull, A. Ochirov and D. O'Connell, A Complete Two-Loop, Five-Gluon Helicity Amplitude in Yang-Mills Theory, JHEP 10 (2015) 064, [1507. 08797].

[14] D. C. Dunbar, G. R. Jehu and W. B. Perkins, Two-loop six gluon all plus helicity amplitude, Phys. Rev. Lett. 117 (2016) 061602, [1605.06351].

[15] J. L. Bourjaily, E. Herrmann, C. Langer and J. Trnka, Building bases of loop integrands, JHEP 11 (2020) 116, [2007.13905].

[16] S. Pozzorini, H. Zhang and M. F. Zoller, Rational Terms of UV Origin at Two Loops, JHEP 05 (2020) 077, [2001.11388].

[17] J.-N. Lang, S. Pozzorini, H. Zhang and M. F. Zoller, Two-Loop Rational Terms in Yang-Mills Theories, JHEP 10 (2020) 016, [2007.03713].

[18] J.-N. Lang, S. Pozzorini, H. Zhang and M. F. Zoller, Two-Loop Rational Terms for Spontaneously Broken Theories, 2107.10288.

[19] D. C. Dunbar and W. B. Perkins, Two-loop five-point all plus helicity Yang-Mills amplitude, Phys. Rev. D 93 (2016) 085029, [1603. 07514].

[20] S. Badger, D. Chicherin, T. Gehrmann, G. Heinrich, J. M. Henn, T. Peraro et al., Analytic form of the full two-loop five-gluon all-plus helicity amplitude, Phys. Rev. Lett. 123 (2019) 071601, [1905.03733].

[21] R. H. Boels, Q. Jin and H. Luo, Efficient integrand reduction for particles with spin, 1802.06761.

[22] T. Peraro and L. Tancredi, Physical projectors for multi-leg helicity amplitudes, JHEP 07 (2019) 114, [1906.03298].

[23] T. Peraro and L. Tancredi, Tensor decomposition for bosonic and fermionic scattering amplitudes, Phys. Rev. D 103 (2021) 054042, [2012 . 00820].

[24] F. Caola, A. Von Manteuffel and L. Tancredi, Diphoton Amplitudes in Three-Loop Quantum Chromodynamics, Phys. Rev. Lett. 126 (2021) 112004, [2011.13946].

[25] F. Caola, A. Chakraborty, G. Gambuti, A. von Manteuffel and L. Tancredi, Three-loop helicity amplitudes for four-quark scattering in massless QCD, 2108.00055. 
[26] L. Chen, A prescription for projectors to compute helicity amplitudes in D dimensions, Eur. Phys. J. C 81 (2021) 417, [1904.00705].

[27] H. A. Chawdhry, M. Czakon, A. Mitov and R. Poncelet, Two-loop leading-colour QCD helicity amplitudes for two-photon plus jet production at the LHC, JHEP 07 (2021) 164, [2103.04319].

[28] H. A. Chawdhry, M. Czakon, A. Mitov and R. Poncelet, Two-loop leading-color helicity amplitudes for three-photon production at the LHC, JHEP 06 (2021) 150, [2012 . 13553].

[29] B. Agarwal, F. Buccioni, A. von Manteuffel and L. Tancredi, Two-loop helicity amplitudes for diphoton plus jet production in full color, 2105.04585.

[30] P. Mastrolia and G. Ossola, On the Integrand-Reduction Method for Two-Loop Scattering Amplitudes, JHEP 11 (2011) 014, [1107.6041].

[31] P. Mastrolia, E. Mirabella, G. Ossola and T. Peraro, Scattering Amplitudes from Multivariate Polynomial Division, Phys. Lett. B 718 (2012) 173-177, [1205 . 7087].

[32] P. Mastrolia, T. Peraro and A. Primo, Adaptive Integrand Decomposition in parallel and orthogonal space, JHEP 08 (2016) 164, [1605.03157].

[33] S. Badger, H. Frellesvig and Y. Zhang, An Integrand Reconstruction Method for Three-Loop Amplitudes, JHEP 08 (2012) 065, [1207.2976].

[34] S. Abreu, J. Dormans, F. Febres Cordero, H. Ita, B. Page and V. Sotnikov, Analytic Form of the Planar Two-Loop Five-Parton Scattering Amplitudes in QCD, JHEP 05 (2019) 084, [1904.00945].

[35] S. Badger, C. Brønnum-Hansen, T. Gehrmann, H. B. Hartanto, J. Henn, N. A. Lo Presti et al., Applications of integrand reduction to two-loop five-point scattering amplitudes in QCD, PoS LL2018 (2018) 006, [1807 . 09709].

[36] S. Abreu, J. Dormans, F. Febres Cordero, H. Ita, M. Kraus, B. Page et al., Caravel: A C++ framework for the computation of multi-loop amplitudes with numerical unitarity, Comput. Phys. Commun. 267 (2021) 108069, [2009. 11957].

[37] S. Abreu, J. Dormans, F. Febres Cordero, H. Ita and B. Page, Analytic Form of Planar Two-Loop Five-Gluon Scattering Amplitudes in QCD, Phys. Rev. Lett. 122 (2019) 082002, [1812.04586].

[38] S. Abreu, B. Page, E. Pascual and V. Sotnikov, Leading-Color Two-Loop QCD Corrections for Three-Photon Production at Hadron Colliders, JHEP 01 (2021) 078, [2010. 15834].

[39] S. Abreu, F. F. Cordero, H. Ita, B. Page and V. Sotnikov, Leading-color two-loop QCD corrections for three-jet production at hadron colliders, JHEP 07 (2021) 095, [2102.13609]. 
[40] S. Abreu, F. F. Cordero, H. Ita, M. Klinkert, B. Page and V. Sotnikov, Leading-Color Two-Loop Amplitudes for Four Partons and a W Boson in QCD, 2110.07541.

[41] S. Badger, C. Brønnum-Hansen, H. B. Hartanto and T. Peraro, Analytic helicity amplitudes for two-loop five-gluon scattering: the single-minus case, JHEP 01 (2019) 186, [1811.11699].

[42] S. Badger, H. B. Hartanto, J. Kryś and S. Zoia, Two-loop leading-colour QCD helicity amplitudes for Higgs boson production in association with a bottom-quark pair at the LHC, 2107.14733.

[43] S. Badger, C. Brønnum-Hansen, D. Chicherin, T. Gehrmann, H. B. Hartanto, J. Henn et al., Virtual QCD corrections to gluon-initiated diphoton plus jet production at hadron colliders, 2106.08664 .

[44] S. Badger, H. B. Hartanto and S. Zoia, Two-Loop QCD Corrections to Wbb- Production at Hadron Colliders, Phys. Rev. Lett. 127 (2021) 012001, [2102.02516].

[45] F. V. Tkachov, A Theorem on Analytical Calculability of Four Loop Renormalization Group Functions, Phys. Lett. B 100 (1981) 65-68.

[46] K. G. Chetyrkin and F. V. Tkachov, Integration by Parts: The Algorithm to Calculate beta Functions in 4 Loops, Nucl. Phys. B 192 (1981) 159-204.

[47] S. Laporta, High precision calculation of multiloop Feynman integrals by difference equations, Int. J. Mod. Phys. A 15 (2000) 5087-5159, [hep-ph/0102033].

[48] P. A. Baikov, K. G. Chetyrkin and J. H. Kühn, Five-Loop Running of the QCD coupling constant, Phys. Rev. Lett. 118 (2017) 082002, [1606.08659].

[49] F. Herzog, B. Ruijl, T. Ueda, J. A. M. Vermaseren and A. Vogt, The five-loop beta function of Yang-Mills theory with fermions, JHEP 02 (2017) 090, [1701. 01404].

[50] P. Maierhöfer, J. Usovitsch and P. Uwer, Kira-A Feynman integral reduction program, Comput. Phys. Commun. 230 (2018) 99-112, [1705.05610].

[51] R. M. Schabinger, A New Algorithm For The Generation Of Unitarity-Compatible Integration By Parts Relations, JHEP 01 (2012) 077, [1111 . 4220].

[52] H. Ita, Two-loop Integrand Decomposition into Master Integrals and Surface Terms, Phys. Rev. D 94 (2016) 116015, [1510.05626].

[53] D. A. Kosower, Direct Solution of Integration-by-Parts Systems, Phys. Rev. D 98 (2018) 025008, [1804.00131].

[54] A. von Manteuffel and R. M. Schabinger, A novel approach to integration by parts reduction, Phys. Lett. B 744 (2015) 101-104, [1406.4513]. 
[55] T. Peraro, Scattering amplitudes over finite fields and multivariate functional reconstruction, JHEP 12 (2016) 030, [1608.01902].

[56] T. Peraro, FiniteFlow: multivariate functional reconstruction using finite fields and dataflow graphs, JHEP 07 (2019) 031, [1905 . 08019].

[57] J. Klappert and F. Lange, Reconstructing rational functions with FireFly, Comput. Phys. Commun. 247 (2020) 106951, [1904.00009].

[58] A. V. Smirnov and F. S. Chuharev, FIRE6: Feynman Integral REduction with Modular Arithmetic, Comput. Phys. Commun. 247 (2020) 106877, [1901. 07808].

[59] J. Klappert, F. Lange, P. Maierhöfer and J. Usovitsch, Integral reduction with Kira 2.0 and finite field methods, Comput. Phys. Commun. 266 (2021) 108024, [2008 . 06494].

[60] E. K. Leĭnartas, Factorization of rational functions of several variables into partial fractions, Soviet Math. (Iz. VUZ) 22 (1978) 35.

[61] A. Raichev, Lěnartas' partial fraction decomposition, 1206.4740.

[62] J. Boehm, M. Wittmann, Z. Wu, Y. Xu and Y. Zhang, IBP reduction coefficients made simple, JHEP 12 (2020) 054, [2008.13194].

[63] M. Heller and A. von Manteuffel, MultivariateApart: Generalized partial fractions, Comput. Phys. Commun. 271 (2022) 108174, [2101.08283].

[64] K.-T. Chen, Iterated path integrals, Bull. Am. Math. Soc. 83 (1977) 831-879.

[65] E. Remiddi and J. A. M. Vermaseren, Harmonic polylogarithms, Int. J. Mod. Phys. A 15 (2000) 725-754, [hep-ph/9905237].

[66] J. Vollinga and S. Weinzierl, Numerical evaluation of multiple polylogarithms, Comput. Phys. Commun. 167 (2005) 177, [hep-ph/0410259].

[67] F. Brown, The Massless higher-loop two-point function, Commun. Math. Phys. 287 (2009) 925-958, [0804.1660].

[68] E. Panzer, Algorithms for the symbolic integration of hyperlogarithms with applications to Feynman integrals, Comput. Phys. Commun. 188 (2015) 148-166, [1403.3385].

[69] A. von Manteuffel, E. Panzer and R. M. Schabinger, On the Computation of Form Factors in Massless QCD with Finite Master Integrals, Phys. Rev. D 93 (2016) 125014, [1510.06758].

[70] M. Bonetti, E. Panzer, V. A. Smirnov and L. Tancredi, Two-loop mixed QCD-EW corrections to $g g \rightarrow H g$, JHEP 11 (2020) 045, [2007.09813].

[71] A. V. Kotikov, Differential equations method: New technique for massive Feynman diagrams calculation, Phys. Lett. B 254 (1991) 158-164. 
[72] E. Remiddi, Differential equations for Feynman graph amplitudes, Nuovo Cim. A 110 (1997) 1435-1452, [hep-th/9711188].

[73] T. Gehrmann and E. Remiddi, Differential equations for two loop four point functions, Nucl. Phys. B 580 (2000) 485-518, [hep-ph/9912329].

[74] J. M. Henn, Multiloop integrals in dimensional regularization made simple, Phys. Rev. Lett. 110 (2013) 251601, [1304 . 1806].

[75] D. Chicherin and V. Sotnikov, Pentagon Functions for Scattering of Five Massless Particles, JHEP 12 (2020) 167, [2009. 07803].

[76] D. Chicherin, V. Sotnikov and S. Zoia, Pentagon Functions for One-Mass Planar Scattering Amplitudes, 2110.10111.

[77] C. G. Papadopoulos, D. Tommasini and C. Wever, The Pentabox Master Integrals with the Simplified Differential Equations approach, JHEP 04 (2016) 078, [1511.09404].

[78] D. D. Canko, C. G. Papadopoulos and N. Syrrakos, Analytic representation of all planar two-loop five-point Master Integrals with one off-shell leg, JHEP 01 (2021) 199, [2009.13917].

[79] C. G. Papadopoulos, Simplified differential equations approach for Master Integrals, JHEP 07 (2014) 088, [1401.6057].

[80] H. A. Chawdhry, M. L. Czakon, A. Mitov and R. Poncelet, NNLO QCD corrections to three-photon production at the LHC, JHEP 02 (2020) 057, [1911. 00479].

[81] S. Kallweit, V. Sotnikov and M. Wiesemann, Triphoton production at hadron colliders in NNLO QCD, Phys. Lett. B 812 (2021) 136013, [2010.04681].

[82] M. Czakon, A. Mitov and R. Poncelet, Next-to-Next-to-Leading Order Study of Three-Jet Production at the LHC, Phys. Rev. Lett. 127 (2021) 152001, [2106.05331].

[83] H. A. Chawdhry, M. Czakon, A. Mitov and R. Poncelet, NNLO QCD corrections to diphoton production with an additional jet at the LHC, 2105.06940.

[84] F. Brown and O. Schnetz, Modular forms in Quantum Field Theory, Commun. Num. Theor Phys. 07 (2013) 293-325, [1304 . 5342].

[85] S. Bloch, M. Kerr and P. Vanhove, Local mirror symmetry and the sunset Feynman integral, Adv. Theor. Math. Phys. 21 (2017) 1373-1453, [1601.08181].

[86] L. Adams, C. Bogner, A. Schweitzer and S. Weinzierl, The kite integral to all orders in terms of elliptic polylogarithms, J. Math. Phys. 57 (2016) 122302, [1607.01571].

[87] J. Ablinger, J. Blümlein, A. De Freitas, M. van Hoeij, E. Imamoglu, C. G. Raab et al., Iterated Elliptic and Hypergeometric Integrals for Feynman Diagrams, J. Math. Phys. 59 (2018) 062305, [1706.01299]. 
[88] E. Remiddi and L. Tancredi, An Elliptic Generalization of Multiple Polylogarithms, Nucl. Phys. B 925 (2017) 212-251, [1709. 03622].

[89] J. Broedel, C. Duhr, F. Dulat and L. Tancredi, Elliptic polylogarithms and iterated integrals on elliptic curves. Part I: general formalism, JHEP 05 (2018) 093, [1712 . 07089].

[90] A. Klemm, C. Nega and R. Safari, The l-loop Banana Amplitude from GKZ Systems and relative Calabi-Yau Periods, JHEP 04 (2020) 088, [1912 . 06201].

[91] S. Borowka, G. Heinrich, S. Jahn, S. P. Jones, M. Kerner, J. Schlenk et al., pySecDec: a toolbox for the numerical evaluation of multi-scale integrals, Comput. Phys. Commun. 222 (2018) 313-326, [1703.09692].

[92] S. Borowka, N. Greiner, G. Heinrich, S. P. Jones, M. Kerner, J. Schlenk et al., Full top quark mass dependence in Higgs boson pair production at NLO, JHEP 10 (2016) 107, [1608.04798].

[93] L. Chen, G. Heinrich, S. P. Jones, M. Kerner, J. Klappert and J. Schlenk, ZH production in gluon fusion: two-loop amplitudes with full top quark mass dependence, JHEP 03 (2021) 125, [2011.12325].

[94] F. Moriello, Generalised power series expansions for the elliptic planar families of Higgs + jet production at two loops, JHEP 01 (2020) 150, [1907. 13234].

[95] M. Hidding, DiffExp, a Mathematica package for computing Feynman integrals in terms of one-dimensional series expansions, Comput. Phys. Commun. 269 (2021) 108125, [2006.05510].

[96] H. Frellesvig, M. Hidding, L. Maestri, F. Moriello and G. Salvatori, The complete set of two-loop master integrals for Higgs + jet production in QCD, JHEP 06 (2020) 093, [1911.06308].

[97] S. Abreu, H. Ita, F. Moriello, B. Page, W. Tschernow and M. Zeng, Two-Loop Integrals for Planar Five-Point One-Mass Processes, JHEP 11 (2020) 117, [2005. 04195].

[98] X. Liu, Y.-Q. Ma and C.-Y. Wang, A Systematic and Efficient Method to Compute Multi-loop Master Integrals, Phys. Lett. B 779 (2018) 353-357, [1711.09572].

[99] C. Brønnum-Hansen and C.-Y. Wang, Contribution of third generation quarks to two-loop helicity amplitudes for W boson pair production in gluon fusion, JHEP 01 (2021) 170, [2009.03742].

[100] C. Brønnum-Hansen and C.-Y. Wang, Top quark contribution to two-loop helicity amplitudes for Z boson pair production in gluon fusion, JHEP 05 (2021) 244, [2101.12095].

[101] X. Liu, Y.-Q. Ma, W. Tao and P. Zhang, Calculation of Feynman loop integration and phase-space integration via auxiliary mass flow, Chin. Phys. C 45 (2021) 013115, [2009.07987]. 
[102] X. Liu and Y.-Q. Ma, Multiloop corrections for collider processes using auxiliary mass flow, 2107.01864. 